\title{
A NEW TYPE OF NONSINGULAR BLACK-HOLE SOLUTION IN GENERAL RELATIVITY
}

\author{
F.R. KLINKHAMER \\ Institute for Theoretical Physics, Karlsruhe Institute of Technology (KIT), \\ 76128 Karlsruhe, Germany \\ frans.klinkhamer@kit.edu
}

\begin{abstract}
Certain exact solutions of the Einstein field equations over nonsimply-connected manifolds are reviewed. These solutions are spherically symmetric and have no curvature singularity. They provide a regularization of the standard Schwarzschild solution with a curvature singularity at the center. Spherically symmetric collapse of matter in $\mathbb{R}^{4}$ may result in these nonsingular black-hole solutions, if quantum-gravity effects allow for topology change near the center or if nontrivial topology is already present as a remnant from a quantum spacetime foam.

Keywords: General relativity; Topology; Exact solutions.

PACS numbers: 04.20.Cv; 02.40.Pc; 04.20.Jb.
\end{abstract}

\section{Introduction}

The main topic of this Brief Review concerns a nonsingular black-hole solution of general relativity, which is closely related to (but not identical with) the standard Schwarzschild solution. ${ }^{[1]}$ It was arrived at by a detour which is rather interesting by itself.

That earlier investigation started from the following simple question: how smooth is space and what quantitative bounds can be set? In order to get a first partial answer to this question, certain Swiss-cheese-type spacetime models were considered, for which the photon propagation can be calculated in the long-wavelength limit. ${ }^{4}[5$ Specifically, these spacetime models have randomly-positioned identical static defects.

The simplest type of defect is obtained as follows: take 3-dimensional Euclidean space, remove the interior of a ball $(r<b)$, and identify antipodal points on the boundary $(r=b)$. The corresponding Swiss-cheese-type model then has two parameters: the defect size $b$ and the average distance $d$ between neighbouring defects. The photon propagation over this spacetime model is described by the isotropic 
modified Maxwell theory with a single Lorentz-violating parameter ${ }^{[5}$

$$
\widetilde{\kappa}_{\mathrm{tr}}=\pi b^{3} / d^{3} .
$$

The isotropic modified Maxwell theory with parameter $\widetilde{\kappa}_{\text {tr }}>0$ [phase velocity of light equal to $\left.\sqrt{\left(1-\widetilde{\kappa}_{\text {tr }}\right) /\left(1+\widetilde{\kappa}_{\text {tr }}\right)} c<c\right]$ and the standard Dirac theory of charged particles [proton limiting velocity equal to $c$ ] give rise to vacuum-Cherenkov radiation. From the observed absence of this nonstandard decay process in the Auger data on ultrahigh-energy cosmic rays, the following two- $\sigma$ upper bound has been obtained! 6

$$
\widetilde{\kappa}_{\mathrm{tr}}<6 \times 10^{-20} .
$$

Two remarks are in order:

(i) Bound (1.2), with the particular identification (1.1), also holds for $b$ and $d$ close to $L_{\text {Planck }} \equiv\left(\hbar G_{N} / c^{3}\right)^{1 / 2} \sim 10^{-35} \mathrm{~m}$.

(ii) The extremely small number on the right-hand side of (1.2) then suggest that, whatever the ultimate theory of quantum gravity may be, this quantum theory must leave practically no defects/wrinkles/ripples on the emerging classical flat spacetime.

All this is quite intriguing, but the particular defect naively embedded in standard Minkowski spacetime does not satisfy the vacuum Einstein field equations (there are delta-function-type curvature singularities ${ }^{7}$ at $r=b$ ) and the same holds for the corresponding Swiss-cheese-type spacetime models. The task, then, is to find a proper defect solution. It turns out that the construction of this nonsingular defect solution ${ }^{8-10}$ produces an interesting spin-off: a black-hole solution without curvature singularity! 11,12

The outline of the present review is as follows. In Sec 2, the relevant topology is discussed. In Sec 3, a nonsingular defect solution is presented, which has no curvature singularity at the center. In Sec 4, the corresponding black-hole solution is discussed, which has an event horizon but still no curvature singularity at the center. In Sec 5, a related black-hole solution is presented with a small but nonzero electric charge. In Sec 6, questions related to physics are discussed. There are also four appendices dealing with technical issues. Two of these appendices contain some important results: the simplest possible description of the nonsingular black-hole solution is given in Appendix C and an essential physics point is discussed in Appendix D A shorter version of this review can be found in Ref. 13.

Let us emphasize, right from the start, that the solutions discussed in this Brief Review are solutions of standard general relativity, no more, no less. The only "new" ingredient is the nontrivial topology, whereas the matter sector is kept entirely standard (e.g., the Maxwell theory of electromagnetism). In this respect, our nonsingular 
black-hole solutions differ from regular solutions obtained with a particular nonlinear extension of electromagnetism. 14] 15 (An extensive list of references on regular black holes can be found in, e.g., Ref. 15.)

\section{Topology}

The four-dimensional spacetime manifold considered is

$$
\widetilde{\mathcal{M}}_{4}=\mathbb{R} \times \widetilde{\mathcal{M}}_{3}
$$

where $\widetilde{\mathcal{M}}_{3}$ is a noncompact, orientable, nonsimply-connected manifold without boundary. Up to a point, $\widetilde{\mathcal{M}}_{3}$ is homeomorphic to the 3 -dimensional real-projective space,

$$
\widetilde{\mathcal{M}}_{3} \simeq \mathbb{R} P^{3}-\{\text { point }\} .
$$

Recall that the 3-dimensional real projective space is topologically equivalent to a 3 -sphere with antipodal points identified. Here, and in the following, the notation is as follows: $\widetilde{\mathcal{M}}$ with tilde stands for a nonsimply-connected manifold [having a nontrivial first homotopy group, $\pi_{1}(\widetilde{\mathcal{M}}) \neq 0$ ] and $\mathcal{M}$ without tilde stands for a simply-connected manifold [having a trivial first homotopy group, $\pi_{1}(\mathcal{M})=0$ ].

For the explicit construction of $\widetilde{\mathcal{M}}_{3}$, we perform local surgery on the 3 dimensional Euclidean space $E_{3}=\left(\mathbb{R}^{3}, \delta_{m n}\right)$. We use the standard Cartesian and spherical coordinates on $\mathbb{R}^{3}$,

$$
\vec{x}=\left(x^{1}, x^{2}, x^{3}\right)=(r \sin \theta \cos \phi, r \sin \theta \sin \phi, r \cos \theta),
$$

with ranges

$$
\begin{aligned}
& x^{m} \in(-\infty,+\infty), \\
& r \in[0, \infty), \quad \theta \in[0, \pi], \quad \phi \in[0,2 \pi) .
\end{aligned}
$$

Now, $\widetilde{\mathcal{M}}_{3}$ is obtained from $\mathbb{R}^{3}$ by removal of the interior of the ball $B_{b}$ with radius $b$ and identification of antipodal points on the boundary $S_{b} \equiv \partial B_{b}$. With point reflection denoted $P(\vec{x})=-\vec{x}$, the 3 -space $\widetilde{\mathcal{M}}_{3}$ is given by

$$
\widetilde{\mathcal{M}}_{3}=\left\{\vec{x} \in \mathbb{R}^{3}:(|\vec{x}| \geq b>0) \wedge(P(\vec{x}) \cong \vec{x} \text { for }|\vec{x}|=b)\right\},
$$

where $\cong$ stands for point-wise identification (Fig. 1).

The next step is to identify appropriate coordinates and a careful analysis has been given in Ref. 7. The standard coordinates of Euclidean 3-space are unsatisfactory, because a single point of $\widetilde{\mathcal{M}}_{3}$ may have different coordinates. For example, $\left(x^{1}, x^{2}, x^{3}\right)=(b, 0,0)$ and $\left(x^{1}, x^{2}, x^{3}\right)=(-b, 0,0)$ correspond to the same point of $\widetilde{\mathcal{M}}_{3}$. 


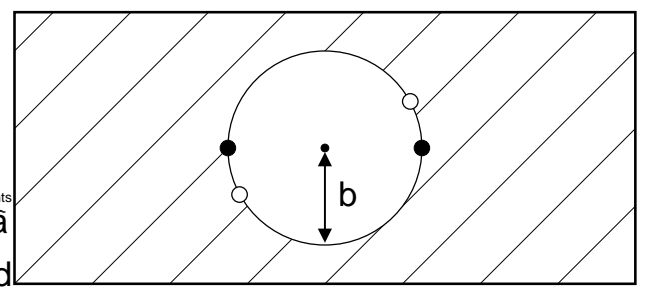

Figure 1. Three-space $\widetilde{\mathcal{M}}_{3}$ obtained by surgery on the 3-dimensional Euclidean space $E_{3}$ : the interior of the ball with radius $b$ is removed and antipodal points on the boundary of the ball are identified (as indicated by open and filled circles). In $E_{3}$, the "long distance" between antipodal points is given by $\pi b$ and the "short distance" by $2 b$.

A relatively simple choice of coordinates for $\widetilde{\mathcal{M}}_{3}$ uses three overlapping charts, each one centered around one of the three Cartesian coordinate axes. The manifold $\widetilde{\mathcal{M}}_{3}$ is now covered by three coordinates charts,

$$
\left(X_{n}, Y_{n}, Z_{n}\right)
$$

for $n=1,2,3$. These coordinates have the following ranges:

$$
\begin{aligned}
& X_{1} \in(-\infty, \infty), \quad Y_{1} \in(0, \pi), \quad Z_{1} \in(0, \pi), \\
& X_{2} \in(0, \pi), \quad Y_{2} \in(-\infty, \infty), \quad Z_{2} \in(0, \pi), \\
& X_{3} \in(0, \pi), \quad Y_{3} \in(0, \pi), \quad Z_{3} \in(-\infty, \infty) .
\end{aligned}
$$

In each chart, there is one radial-type coordinate with infinite range, one polar-type angular coordinate of finite range, and one azimuthal-type angular coordinate of finite range. The charts overlap: see Fig. 2 for a sketch and Appendix A for further details.

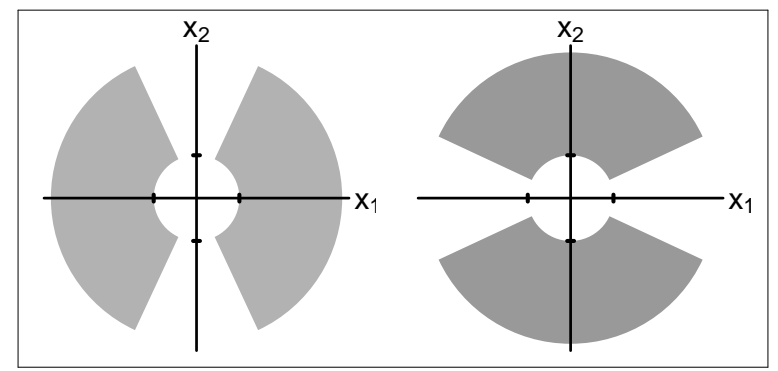

Figure 2. Slice $x_{3}=0$ of the manifold $\widetilde{\mathcal{M}}_{3}$ with the domains of the chart- 1 coordinates (left) and the chart- 2 coordinates (right). The tick marks on the $x_{1}$ and $x_{2}$ axes correspond to the values $\pm b$; see Fig. 1 The 3-dimensional domains are obtained by revolution around the $x_{1}$-axis (left) or the $x_{2}$-axis (right). The domain of the chart-3 coordinates is defined similarly. 
In the following, we will explicitly work with only one coordinate chart, which we take to be (2.5b). Moreover, we will drop the suffix ' 2 ' on these coordinates:

$$
(T, X, Y, Z) \equiv\left(T, X_{2}, Y_{2}, Z_{2}\right),
$$

where the time coordinate $T$ has been added in order to describe part of the spacetime manifold $\widetilde{\mathcal{M}}_{4}$.

Two final remarks are in order. First, the previous view of $\widetilde{\mathcal{M}}_{3}$ starting from Euclidean 3-space (Fig. 1) may be partly misleading if trajectories through the defect are considered. Consider a particular slice of $\mathbb{R} P^{3}$ which gives $\mathbb{R} P^{2}$. This manifold $\mathbb{R} P^{2}$, the real projective plane, can be immersed in $\mathbb{R}^{3}$ as Boy's surface (Fig. 3). Now, one particular trajectory may not look smooth at all in the view based on the 2-plane with surgery (Fig. 4-left) but is manifestly smooth if viewed as a curve over Boy's surface (Fig. 4-right).

Second, general relativity is all about coordinate independence of the physics, but a pre-requisite is to have at least one set of proper coordinates covering the manifold. Precisely this pre-requisite has been established with the coordinates (2.4).

\section{Nonsingular Defect Solution}

As explained in the Introduction, our goal is to find a genuine solution of the vacuum Einstein field equations, with a parameter $b>0$ and topology as suggested by the sketch in Fig. 11 and detailed in Sec. 2,

A spherically symmetric Ansatz for the metric over $\widetilde{\mathcal{M}}_{4}$ is given by the line element

$$
\begin{aligned}
\left.d s^{2}\right|_{\text {chart-2 }}= & -\exp [2 \widetilde{\nu}(W)] d T^{2}+\exp [2 \widetilde{\lambda}(W)] d Y^{2} \\
& +W\left(d Z^{2}+\sin ^{2} Z d X^{2}\right) \\
\left.W\right|_{\text {chart-2 }} \equiv & \zeta^{2} \\
\left.\zeta\right|_{\text {chart-2 }} \equiv & \sqrt{b^{2}+Y^{2}}
\end{aligned}
$$

with a length parameter $b>0$. In (3.1), only the coordinates of the $n=2$ chart (2.5b) have been shown, dropping the suffix ' 2 ' on these coordinates. Recall that the 'radial' coordinate $Y$ takes values in $(-\infty, \infty)$, with positive values of $Y$ on one side of the defect and negative values on the other side.

With this Ansatz and units $G_{N}=c=1$, the following exact solution ${ }^{8}$ of the vacuum Einstein field equations is obtained:

$$
\begin{aligned}
& \exp [2 \widetilde{\nu}(W)]=1-2 M / \sqrt{W}, \\
& \exp [2 \widetilde{\lambda}(W)]=\frac{1-b^{2} / W}{1-2 M / \sqrt{W}},
\end{aligned}
$$




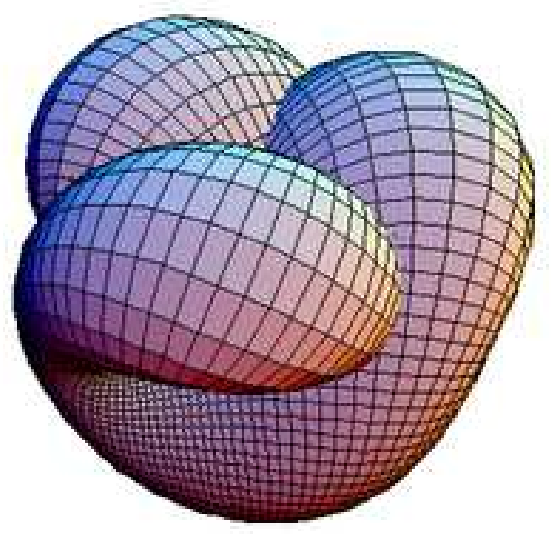

Figure 3. Boy's surface immersed in $\mathbb{R}^{3}$. Boy's surface is homeomorphic to the real projective plane, $\mathbb{R} P^{2}$. Figure obtained by use of the Bryant-Kusner parametrization of $\mathbb{R} P^{2}$ and the computer program MATHEMATICA $[16$
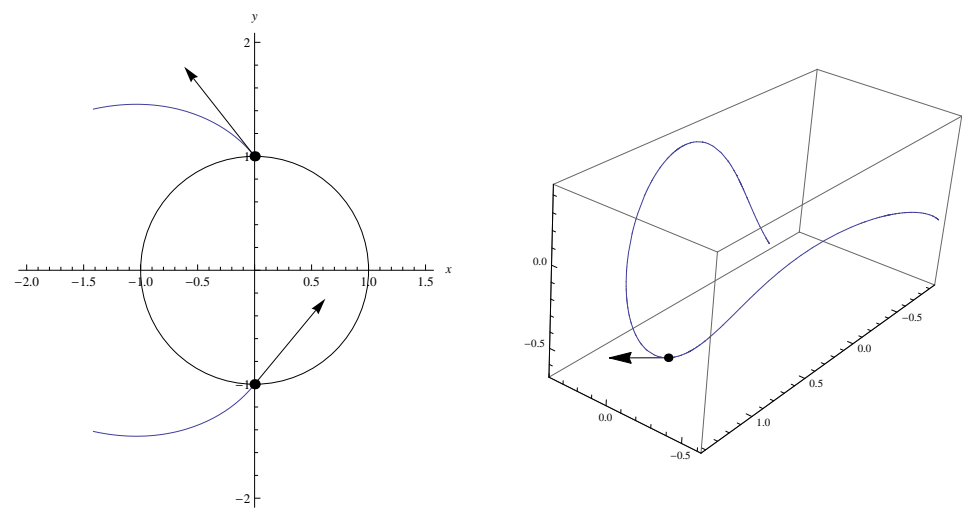

Figure 4. Trajectory shown in two representations of $\mathbb{R} P^{2}$. Left: trajectory shown in the 2-plane with surgery. Right: trajectory shown over Boy's surface (the surface itself is not displayed). Figures by courtesy of M. Schwarz $]^{7}$

in terms of a further parameter $M$ taken to obey

$$
2 M<b,
$$

so that the metric component (3.2a) does not vanish over the whole range of $Y$. The solution for $M=0$ will be seen to correspond to the flat spacetime with nontrivial topology (2.1a) [see Appendix D for a 'blemish' of this spacetime]. Later, we will comment on the Schwarzschild-like structure apparent in (3.2).

The resulting Riemann curvature tensor is finite over the whole manifold, in- 
cluding the defect core at $W=b^{2}$ :

$$
\begin{aligned}
& R_{Y T Y}^{T}=\frac{2 M\left(W-b^{2}\right)}{W^{2}(\sqrt{W}-2 M)}, \\
& R_{Z T Z}^{T}=-M / \sqrt{W}, \\
& R_{Z Y Z}^{Y}=-M / \sqrt{W}, \\
& R_{X Z X}^{Z}=(\sin Z)^{2} 2 M / \sqrt{W} .
\end{aligned}
$$

More importantly, also the Kretschmann curvature scalar,

$$
K \equiv R_{\mu \nu \rho \sigma} R^{\mu \nu \rho \sigma}=48 \frac{M^{2}}{W^{3}},
$$

remains finite over the whole of $\widetilde{\mathcal{M}}_{4}$, because $W \geq b^{2}>0$. Some details of the calculation of the Riemann curvature tensor are presented in Appendix B.

Radial geodesics can be readily calculated ${ }^{[8}$ It is even possible to obtain explicit solutions for the special case $M=0$ (flat spacetime). Up to arbitrary time shifts, the radial geodesics are then given in terms of two real constants $A$ and $B$ (with $B$ taken positive):

$$
\begin{aligned}
& Y(T)=A b, \\
& Y(T)=\left\{\begin{array}{lll} 
\pm \sqrt{(B T)^{2}+2 B T} b & \text { for } \quad T \geq 0, \\
\mp \sqrt{(B T)^{2}-2 B T} b & \text { for } \quad T<0,
\end{array}\right.
\end{aligned}
$$

where the lower entries in front of the square roots on the right-hand side of (3.6b) correspond to a negative asymptotic velocity and the upper entries to a positive asymptotic velocity (Fig. [5).

The spacetime manifold $\widetilde{\mathcal{M}}_{4}$ with topology (2.1) is covered by three coordinate charts, as explained in Sec. 2 and Appendix A. Using the results (3.1) and (3.2)

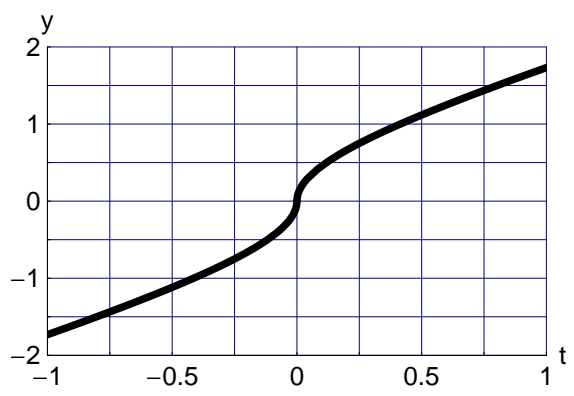

Figure 5. Radial geodesic 3.6b in terms of dimensionless coordinates $y$ and $t$. 
for the chart-2 coordinates (temporarily restoring the suffix ' 2 '), the respective line elements are

$$
\begin{aligned}
\left.d s^{2}\right|_{\text {chart- } 1}= & -\left(1-\frac{2 M}{\sqrt{b^{2}+\left(X_{1}\right)^{2}}}\right) d T^{2} \\
& +\left(1-\frac{2 M}{\sqrt{b^{2}+\left(X_{1}\right)^{2}}}\right)^{-1} \frac{\left(X_{1}\right)^{2}}{b^{2}+\left(X_{1}\right)^{2}}\left(d X_{1}\right)^{2} \\
& +\left(b^{2}+\left(X_{1}\right)^{2}\right)\left(\left(d Z_{1}\right)^{2}+\left(\sin Z_{1}\right)^{2}\left(d Y_{1}\right)^{2}\right), \\
\left.d s^{2}\right|_{\text {chart-2 }}= & -\left(1-\frac{2 M}{\sqrt{b^{2}+\left(Y_{2}\right)^{2}}}\right) d T^{2} \\
& +\left(1-\frac{2 M}{\sqrt{b^{2}+\left(Y_{2}\right)^{2}}}\right)^{-1} \frac{\left(Y_{2}\right)^{2}}{b^{2}+\left(Y_{2}\right)^{2}}\left(d Y_{2}\right)^{2} \\
& +\left(b^{2}+\left(Y_{2}\right)^{2}\right)\left(\left(d Z_{2}\right)^{2}+\left(\sin Z_{2}\right)^{2}\left(d X_{2}\right)^{2}\right), \\
\left.d s^{2}\right|_{\text {chart-3 }}= & \left(1-\frac{2 M}{\sqrt{b^{2}+\left(Z_{3}\right)^{2}}}\right)^{-1} d T^{2} \\
& +\left(1-\frac{2 M}{\sqrt{b^{2}+\left(Z_{3}\right)^{2}}}\right)^{\frac{\left(Z_{3}\right)^{2}}{b^{2}+\left(Z_{3}\right)^{2}}\left(d Z_{3}\right)^{2}} \\
& +\left(b^{2}+\left(Z_{3}\right)^{2}\right)\left(\left(d Y_{3}\right)^{2}+\left(\sin Y_{3}\right)^{2}\left(d X_{3}\right)^{2}\right) .
\end{aligned}
$$

This completes the discussion of the nonsingular defect solution with parameters $b>0$ and $2 M<b$.

\section{Nonsingular Black-Hole Solution}

The defect metric (3.1)-(3.2) can be seen to take precisely the form of the standard exterior-region Schwarzschild solution, in line with Birkhoff's theorem. ${ }^{2 \mid 3}$ If $Y^{2}+$ $b^{2}$ is identified with $r^{2}$, the line element from (3.1) and (3.2) contains the term $\left(1-b^{2} / W\right) d Y^{2}=Y^{2} /\left(b^{2}+Y^{2}\right) d Y^{2}=d r^{2}$ and the line element indeed takes the standard Schwarzschild form.

Starting from this observation, we can obtain a black-hole solution ${ }^{11}$ for the topology

$$
\begin{aligned}
& \widetilde{\mathcal{M}}_{4}=\mathbb{R} \times \widetilde{\mathcal{M}}_{3}, \\
& \widetilde{\mathcal{M}}_{3} \simeq \mathbb{R} P^{3}-\{\text { point }\},
\end{aligned}
$$

with parameters:

$$
2 M>b>0 .
$$


For this new black-hole solution (and another one described in Sec. 5), the curvature singularity will be eliminated by a spacetime defect, i.e., a "hole" in spacetime.

The solution will be presented in terms of two sets of chart- 2 coordinates, one set appropriate to the spacetime defect and another set further out (here, taken to be Kruskal-Szekeres-type coordinates ${ }^{17}[18)$. But it is also possible to use only one set of chart-2 coordinates; see Appendix C for details.

At and near the spacetime defect, the metric is given by

$$
\begin{aligned}
\left.d s^{2}\right|_{\text {chart-2 }} ^{(b \leq \zeta<2 M)}= & +\left(\frac{2 M}{\zeta}-1\right) d T^{2}-\left(\frac{2 M}{\zeta}-1\right)^{-1} \frac{Y^{2}}{\zeta^{2}} d Y^{2} \\
& +\zeta^{2}\left(d Z^{2}+\sin ^{2} Z d X^{2}\right) \\
\left.\zeta(Y)\right|_{\text {chart-2 }}= & \sqrt{b^{2}+Y^{2}} .
\end{aligned}
$$

Further out, the metric is given by

$$
\begin{aligned}
\left.d s^{2}\right|_{\text {chart-2 }} ^{(b<\zeta)}= & 32 M^{3} \frac{\exp [-\zeta /(2 M)]}{\zeta}\left(-d V^{2}+d U^{2}\right) \\
& +\zeta^{2}\left(d Z^{2}+\sin ^{2} Z d X^{2}\right),
\end{aligned}
$$

with $\zeta$ written in terms of Kruskal-Szekeres-type coordinates $U, V \in \mathbb{R}$ by use of the principal branch of the Lambert $W$-function:

$$
\left.\zeta(U, V)\right|_{\text {chart-2 }}=2 M\left(1+W_{0}\left[\frac{U^{2}-V^{2}}{e}\right]\right) .
$$

Recall that the principal branch of the Lambert $W$-function, $W_{0}[z]$, gives the principal solution for $w$ in $z=w \exp [w]$ and that $W_{0}[x]$ is real for $x \in \mathbb{R}$ and $x \geq-1 / e$. The radial coordinate $\zeta$ from (4.4b) reduces to the following expression:

$$
\left.\zeta(U, V)\right|_{\text {chart-2 }}=\sqrt{b^{2}+Y^{2}},
$$

by use of the coordinate transformations ${ }^{2 \mid 3}$

$$
\begin{aligned}
& \left.U\right|_{\text {chart-2 }}=\left\{\begin{array}{l}
\left(\frac{\sqrt{Y^{2}+b^{2}}}{2 M}-1\right)^{1 / 2} \exp \left[\frac{\sqrt{Y^{2}+b^{2}}}{4 M}\right] \cosh \left[\frac{T}{4 M}\right], \\
\left(1-\frac{\sqrt{Y^{2}+b^{2}}}{2 M}\right)^{1 / 2} \exp \left[\frac{\sqrt{Y^{2}+b^{2}}}{4 M}\right] \sinh \left[\frac{T}{4 M}\right],
\end{array}\right. \\
& \left.V\right|_{\text {chart-2 }}= \begin{cases}\left(\frac{\sqrt{Y^{2}+b^{2}}}{2 M}-1\right)^{1 / 2} \exp \left[\frac{\sqrt{Y^{2}+b^{2}}}{4 M}\right] \sinh \left[\frac{T}{4 M}\right], \\
\left(1-\frac{\sqrt{Y^{2}+b^{2}}}{2 M}\right)^{1 / 2} \exp \left[\frac{\sqrt{Y^{2}+b^{2}}}{4 M}\right] \cosh \left[\frac{T}{4 M}\right],\end{cases}
\end{aligned}
$$


with top entries for the exterior region $\left(\sqrt{Y^{2}+b^{2}}>2 M\right)$ and bottom entries for the interior region $\left(\sqrt{Y^{2}+b^{2}}<2 M\right)$.

The Riemann tensor takes the same form as for the defect solution, see (3.4). The Ricci tensor and Ricci scalar vanish identically. Hence, the vacuum Einstein field equations are solved. Furthermore, the Kretschmann scalar is given by

$$
K \equiv R_{\mu \nu \rho \sigma} R^{\mu \nu \rho \sigma}=48 \frac{M^{2}}{\zeta^{6}},
$$

with $\zeta^{2}=b^{2}+Y^{2}$ over the chart-2 domain, both for the inner metric (4.3) and the outer metric (4.4), and similarly over the two other domains. The Kretschmann scalar remains finite because $b>0$.

Recall that the standard Schwarzschild-Kruskal-Szekeres solution 1$] 17][18$ with topology

$$
\mathcal{M}_{\mathrm{SKS}}=\mathbb{R}^{2} \times S^{2}
$$

has a physical singularity for $r \rightarrow 0$, as shown by the divergence of the Kretschmann scalar,

$$
\left.\left.K\right|_{\mathrm{SKS}} \equiv R_{\mu \nu \rho \sigma} R^{\mu \nu \rho \sigma}\right|_{\mathrm{SKS}}=48 \frac{M^{2}}{r^{6}} .
$$

The comparison of (4.6) for $b \neq 0$ and (4.8) makes clear that the solution (4.3)(4.4) over $\mathbb{R} \times \widetilde{\mathcal{M}}_{3}$ may be considered to be a regularized version of the standard Schwarzschild solution over $\mathbb{R}^{+} \times \mathbb{R} \times S^{2}$, with the curvature singularity eliminated by a spacetime defect, i.e., a "hole" in spacetime.

The coordinate $T$ and the coordinate $Y$ are, respectively, spacelike and timelike for the inner metric (4.3). This behavior is analogous to what happens for the standard Schwarzschild solution ${ }^{3}$ Note, however, that the timelike coordinate $Y$ of the inner metric (4.3) ranges from $-\infty$ to $+\infty$, unlike the usual radial coordinate $r$. Moreover, this timelike coordinate $Y$ is part of a topologically nontrivial manifold $\widetilde{\mathcal{M}}_{3}$. This gives rise to the presence of closed time-like curves (CTCs). These CTCs imply all possible horrors, but, classically, these horrors remain confined within the Schwarzschild horizon. Whether or not CTCs in the interior region are physically acceptable depends on the behavior of the matter fields a

\section{Nonsingular Black-Hole Solution with Electric Charge}

The problematic closed timelike curves of the modified Schwarzschild solution (4.3) trace back to the fact that the original singularity was spacelike. But it is wellknown that the singularity of the standard Reissner-Nordström (RN) solution 21.22

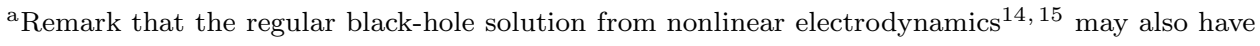
problems with causality: absence of a global Cauchy surface (i.e., incomplete predictability of the future) and possible lack of microcausality for the electromagnetic fields (cf. Refs. [19 20). 


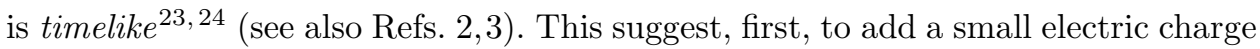
and, then, to modify the resulting $\mathrm{RN}$ solution in order to arrive at a nonsingular solution. 12

Consider, then, spherically symmetric solutions of the Einstein field equations,

$$
R_{\mu}{ }^{\nu}(x)-\frac{1}{2} R(x) \delta_{\mu}{ }^{\nu}=8 \pi T_{\mu}{ }^{\nu}(x),
$$

where the energy-momentum tensor $T_{\mu}{ }^{\nu}$ is set equal to a prescribed energymomentum tensor $\Theta_{\mu}{ }^{\nu}$ (using spherical coordinates),

$$
T_{\mu}{ }^{\nu}(t, r, \theta, \phi)=\Theta_{\mu}{ }^{\nu}(t, r, \theta, \phi) \equiv \frac{Q^{2}}{8 \pi r^{4}} D_{\mu}{ }^{\nu}(t, r, \theta, \phi),
$$

in terms of the traceless diagonal matrix

$$
D_{\mu}{ }^{\nu}(t, r, \theta, \phi) \equiv\left\{\begin{array}{cl}
-1 & \text { for } \mu=\nu \in\{t, r\} \\
+1 & \text { for } \mu=\nu \in\{\theta, \phi\} \\
0 & \text { otherwise }
\end{array}\right.
$$

This particular $\Theta_{\mu}{ }^{\nu}$ corresponds to the energy-momentum tensor of a Coulombtype electric field. (It is also possible to deal with the coupled Einstein and Maxwell equations, but we simplify the discussion by use of a fixed Coulomb-type energymomentum tensor.)

The standard Reissner-Nordström solution in the exterior region has a metric given by the following line element:

$$
\begin{aligned}
\left.d s^{2}\right|_{\mathrm{RN}} ^{\left(r>r_{+}\right)}= & -\left(1-\frac{2 M}{r}+\frac{Q^{2}}{r^{2}}\right) d t^{2}+\left(1-\frac{2 M}{r}+\frac{Q^{2}}{r^{2}}\right)^{-1} d r^{2} \\
& +r^{2}\left(d \theta^{2}+\sin ^{2} \theta d \phi^{2}\right)
\end{aligned}
$$

with coordinates $t \in \mathbb{R}, r>r_{+} \equiv M+\sqrt{M^{2}-Q^{2}}, \theta \in[0, \pi], \phi \in[0,2 \pi)$. Here, $M$ can be interpreted as the mass of the central object and $Q$ as its electric charge.

The corresponding nonsingular solution in terms of an effective radial coordinate $\zeta$ will be seen to have a further length parameter $b$. The three parameters of the solution are taken to be related as follows:

$$
\begin{aligned}
& 0<|Q|<M, \\
& 0<b<\zeta_{-},
\end{aligned}
$$

with definitions

$$
\zeta_{ \pm} \equiv M \pm \sqrt{M^{2}-Q^{2}} .
$$

Note that, for the classical theory, the electric charge $|Q|$ can be arbitrarily small, as long as the charge $Q$ remains nonzero and the length $b$ is sufficiently small, $b<\zeta_{-} \sim Q^{2} /(2 M)$. [A different choice of parameters is considered in Footnote $\mathrm{C}$ of Appendix C] 
For the construction of the nonsingular solution with parameters (5.3), we refer to Carter's original article ${ }^{24}$ for the standard Reissner-Nordström solution and follow the same modification procedure used in Sec. 4 for the Schwarzschild solution. As we are primarily interested in the removal of the curvature singularity, we focus on the spacetime region III $\left(0 \leq \zeta<\zeta_{-}\right)$. No essential change occurs for the spacetime regions I and II $\left(\zeta \geq \zeta_{-}>0\right)$, because they do not reach the singularity at $\zeta=0$.

The region-III metric with the defect at $\zeta=b$ included is then found to have the following line element:

$$
\begin{aligned}
\left.d s^{2}\right|_{\text {chart-2 }} ^{\left(b \leq \zeta<\zeta_{-}\right)}= & -\left(1-\frac{2 M}{\zeta}+\frac{Q^{2}}{\zeta^{2}}\right) d T^{2}+\left(1-\frac{2 M}{\zeta}+\frac{Q^{2}}{\zeta^{2}}\right)^{-1} \frac{Y^{2}}{\zeta^{2}} d Y^{2} \\
& +\zeta^{2}\left(d Z^{2}+\sin ^{2} Z d X^{2}\right) \\
\left.\zeta\right|_{\text {chart-2 }}= & \sqrt{b^{2}+Y^{2}}
\end{aligned}
$$

From the standard analysis, $\frac{2[3}{2}$ it follows that the singularities at $\zeta=\zeta_{ \pm}$in (5.5a) can be removed by coordinate transformations.

Note that (5.5a) takes precisely the form of the original Reissner-Nordström metric (5.2) if $\left(Y^{2} / \zeta^{2}\right) d Y^{2}$ is replaced by $d \zeta^{2}$ according to (5.5b). But, as emphasized before, the crucial point here is the appearance of the coordinate $Y \in(-\infty, \infty)$ of the nonsimply-connected manifold $\widetilde{\mathcal{M}}_{3}$. In addition, there are now radial geodesics passing through $Y=0$, as explained in the penultimate paragraph of Sec. 3 ,

The metric (5.5) solves the Einstein field equations (5.1a) for a prescribed energymomentum tensor $\Theta_{\mu}{ }^{\nu}(T, X, Y, Z)$ of the diagonal form (5.1b) with $1 / r^{4}$ replaced by $1 / \zeta^{4}=1 /\left(b^{2}+Y^{2}\right)^{2}$.

The spacetime from the metric (5.5), extended to all charts, corresponds to a noncompact, orientable, nonsimply-connected manifold without boundary. This spacetime has the topology

$$
\begin{aligned}
\left.\widetilde{\mathcal{M}}\right|_{\text {mod-RN }} & =\mathbb{R} \times \widetilde{\mathcal{M}}_{3}, \\
\widetilde{\mathcal{M}}_{3} & \simeq \mathbb{R} P^{3}-\{\text { point }\},
\end{aligned}
$$

where 'mod-RN' stands for the modified Reissner-Nordström solution and $\mathbb{R} P^{3}$ is the 3-dimensional real projective space (topologically equivalent to a 3 -sphere with antipodal points identified).

The Kretschmann curvature scalar over the manifold (5.6) is given by

$$
\left.\left.K\right|_{\text {mod-RN }} \equiv R_{\mu \nu \rho \sigma} R^{\mu \nu \rho \sigma}\right|_{\text {mod-RN }}=\frac{8\left(6 M^{2} \zeta^{2}-12 M Q^{2} \zeta+7 Q^{4}\right)}{\zeta^{8}}
$$

which remains finite because $\zeta>0$ for $b>0$. For fixed values of $M$ and $Q$ obeying condition (5.3a), $K(\zeta)$ drops monotonically with $\zeta$. This fact allows for an operational definition of $b$ from the maximum value of $K$. (The operational definitions of 
$M$ and $Q$ rely, for example, on the asymptotic $\zeta \rightarrow \infty$ behavior of the metric and electromagnetic fields.)

The main result of this section is that the factor $\mathbb{R}$ in (5.6a) corresponds to the timelike direction of the metric (5.5), making for a spacelike hypersurface $\widetilde{\mathcal{M}}_{3}$ in the spacetime region III. In turn, this observation implies the absence of closed timelike curves. Recall that the spacetime regions II $\left(\zeta_{-}<\zeta<\zeta_{+}\right)$and I $\left(\zeta>\zeta_{+}\right)$do not reach the $\zeta=b$ surface where antipodal points are identified (cf. Fig. 11).

Mathematically speaking, the nonsingular black-hole solution (5.5) with parameters (5.3) can be viewed as a "regularization" of the singular Reissner-Nordström solution $\mathrm{b}$

\section{Discussion}

Apart from the mathematical interest of having a new type of exact solution of the Einstein field equations, these nonsingular solutions (with or without electric charge) may also appear in a physical context. Let us focus on the charged nonsingular solution (5.5) and perform the following Gedankenexperiment.

Start from a nearly flat spacetime with a trivial topology $\mathbb{R}^{4}$ and a metric approximately equal to the Minkowski metric. Next, arrange for a large amount of matter with total mass $\bar{M}$ and with vanishing net charge $\bar{Q}=0$ to collapse in a spherically symmetric way. Within the realm of classical Einstein gravity, we expect to end up with the singular Schwarzschild solution. But, very close to the final curvature singularity, something else may happen due to quantum effects.

Consider a precursor mass $\overline{\Delta M} \sim \hbar /(b c) \ll \bar{M}$ and use typical curvature values from the expressions (4.6) and (4.8) for the Kretschmann scalar. Then, the local spacetime integral of the action density related to the standard Schwarzschild solution differs from that related to (5.5) for $Q^{2} \ll M^{2}$ by an amount $\lesssim \hbar$. As argued by Wheeler in particular, the local topology of the manifold may now change by a quantum jump if $b$ is sufficiently close to the length scale $L_{\text {Planck }} \equiv\left(\hbar G_{N} / c^{3}\right)^{1 / 2}$. In addition, the strong gravitational fields may lead to electron-positron pair creation, possibly with one charge expelled towards spatial infinity.

These two quantum processes combined may result in a transition from the simply-connected manifold $\mathbb{R}^{4}$ without localized charge $Q$ to the nonsimplyconnected manifold $\widetilde{\mathcal{M}}_{4}$ with localized charge $Q= \pm\left|Q_{\text {electron }}\right| \equiv \pm e$. Hence, if the transition amplitude between the different topologies is nonzero for appropriate matter content, quantum mechanics can operate a change between the classi-

\footnotetext{
${ }^{\mathrm{b}}$ It is well-known that the standard Reissner-Nordström metric 5.2 with $M=0$ has a naked singularity. Not so for the metric (5.5) with $M=0$. This may provide new impetus to discussions of elementary particles (rotation/spin neglected, for the moment) being interpreted as tiny spacetime structures (cf. Sec. 5.2 of Ref. 25). Note that macroscopic naked singularities (and their regulated versions) have distinctive gravitational-lensing characteristics 26
} 
cal Schwarzschild solution and the classical solution (5.5) with $\bar{Q}= \pm e$ and an additional charge $\mp e$ at infinity. In this way, the curvature singularity would be eliminated, but not at the price of introducing closed timelike curves.

It is also possible to present an alternative scenario without topology change. Now, the spherical collapse of matter is assumed to occur in Minkowski spacetime with a relatively sparse sprinkling of massless static defects (each one given by the solution of Sec. 2 with $M=0$ and $b>0$ ). Then, the precursor mass $\overline{\Delta M}$ selects one of the available defect cores and increases its mass $(M=0 \rightarrow \overline{\Delta M} \rightarrow \bar{M})$, possibly giving it also a charge by electron-positron pair creation with one charge expelled to infinity. Again, the curvature singularity would be eliminated, while avoiding closed timelike curves.

Many questions remain, the most important of which are the following:

(i) Are these regularized Schwarzschild solutions really acceptable, both mathematically and physically?

(ii) Are there perhaps other surprises from this regularization, in a way reminiscent of the anomalies of quantum field theory?

(iii) Where does the matter go, is it distributed over a thin shell with $\zeta \in[b, b+\Delta b)$ for positive $\Delta b$ ?

(iv) Does realistic collapse occur with or without topology change?

These are obviously difficult questions. A partial answer to the first question appears in Appendix D, where a particular characteristic of the regularized spacetime is discussed. Further progress on all questions can perhaps be made by direct investigations of the matter sector [cf. Ref. 9 for a nonsingular defect solution with an $S O(3)$ Skyrmion field].

\section{Acknowledgments}

It is a pleasure to thank C. Lämmerzahl for discussions on elementary flatness and A. Macias for pointing out Ref. 14.

\section{Appendix A. Coordinate Charts}

The three coordinate charts of the 3 -manifold $\widetilde{\mathcal{M}}_{3}$ with topology (2.1b) were briefly discussed in Sec. 2, Further details will be given in this appendix.

A relatively simple covering of $\widetilde{\mathcal{M}}_{3}$ uses three charts of coordinates, labeled by $n=1,2,3$. Each chart covers and surrounds part of one of the three Cartesian coordinate axes but does not intersect the other two axes. For example, the $n=1$ coordinate chart covers and surrounds the $\left|x^{1}\right| \geq b$ parts of the $x^{1}$ coordinate axis but does not intersect the $x^{2}$ and $x^{3}$ axes. The domains of the chart- 1 coordinates 
consist of two 'wedges,' on both sides of the defect and pierced by the $x^{1}$ axis; see Fig. 2-left.

These coordinates are denoted $\left(X_{n}, Y_{n}, Z_{n}\right)$, for $n=1,2,3$, and their ranges were already given by (2.5) of the main text. In order to describe the interrelation of the coordinates $\left(X_{n}, Y_{n}, Z_{n}\right)$ in the overlap regions, we express them in terms of the coordinates of the 3-dimensional Euclidean space $E_{3}$. For the latter, we use both standard and nonstandard spherical coordinates. The standard spherical coordinates $(r, \theta, \phi)$ are defined by

$$
\left(x^{1}, x^{2}, x^{3}\right)=(r \sin \theta \cos \phi, r \sin \theta \sin \phi, r \cos \theta),
$$

with $r \geq 0, \theta \in[0, \pi]$, and $\phi \in[0,2 \pi)$. The nonstandard spherical coordinates $(r, \vartheta, \varphi)$ are defined by

$$
\left(x^{1}, x^{2}, x^{3}\right)=(r \sin \vartheta \sin \varphi, r \cos \vartheta, r \sin \vartheta \cos \varphi),
$$

with $r \geq 0, \vartheta \in[0, \pi]$, and $\varphi \in[0,2 \pi)$.

Now, the chart- 1 and chart-2 coordinates over the appropriate regions (wedges) of $\widetilde{M}_{3}$ are given by

$$
\begin{aligned}
& X_{1}=\left\{\begin{array}{lll}
r-b & \text { for } & \cos \phi>0, \\
b-r & \text { for } & \cos \phi<0,
\end{array}\right. \\
& Y_{1}=\left\{\begin{array}{lll}
\phi-\pi / 2 & \text { for } & \pi / 2<\phi<3 \pi / 2, \\
\phi-3 \pi / 2 & \text { for } & 3 \pi / 2<\phi<2 \pi, \\
\phi+\pi / 2 & \text { for } & 0 \leq \phi<\pi / 2,
\end{array}\right. \\
& Z_{1}=\left\{\begin{array}{lll}
\theta & \text { for } & \cos \phi>0, \\
\pi-\theta & \text { for } & \cos \phi<0,
\end{array}\right.
\end{aligned}
$$

and

$$
\begin{gathered}
X_{2}=\left\{\begin{array}{lll}
\phi & \text { for } & 0<\phi<\pi, \\
\phi-\pi & \text { for } & \pi<\phi<2 \pi,
\end{array}\right. \\
Y_{2}=\left\{\begin{array}{lll}
r-b & \text { for } & 0<\phi<\pi, \\
b-r & \text { for } & \pi<\phi<2 \pi,
\end{array}\right. \\
Z_{2}=\left\{\begin{array}{lll}
\theta & \text { for } & 0<\phi<\pi, \\
\pi-\theta & \text { for } & \pi<\phi<2 \pi,
\end{array}\right.
\end{gathered}
$$

in terms of the standard spherical coordinates (A.1).

The $n=3$ chart requires coordinates of $E_{3}$ that are regular on the Cartesian $x^{3}$ axis. The chart- 3 coordinates over the relevant regions (wedges) of $\widetilde{M}_{3}$ are then 
given by

$$
\begin{aligned}
& X_{3}=\left\{\begin{array}{lll}
\varphi-\pi / 2 & \text { for } & \pi / 2<\varphi<3 \pi / 2, \\
\varphi-3 \pi / 2 & \text { for } & 3 \pi / 2<\varphi<2 \pi, \\
\varphi+\pi / 2 & \text { for } & 0 \leq \varphi<\pi / 2,
\end{array}\right. \\
& Y_{3}=\left\{\begin{array}{lll}
\vartheta & \text { for } & \cos \varphi>0, \\
\pi-\vartheta & \text { for } & \cos \varphi<0,
\end{array}\right. \\
& Z_{3}=\left\{\begin{array}{lll}
r-b & \text { for } & \cos \varphi>0, \\
b-r & \text { for } & \cos \varphi<0,
\end{array}\right.
\end{aligned}
$$

in terms of the nonstandard spherical coordinates A.2.

It can be verified that these three sets of coordinates $\left(X_{n}, Y_{n}, Z_{n}\right)$ are invertible and infinitely-differentiable functions of each other in the overlap regions. Moreover, the manifold satisfies the Hausdorff property, i.e., two distinct points can each be surrounded by open sets, so that these two open sets do not overlap (see Ref. 7 for details).

\section{Appendix B. Riemann Curvature Tensor}

In this appendix, we calculate the Riemann curvature tensor for the nonsingular defect metric (3.1)-(3.2) by using two deformations of the metric. The deformed metrics never vanish in the domains over which they are defined and are directly invertible. The parameters of the defect solution are $b>0$ and $2 M<b$, but the same analysis applies to the case $2 M>b>0$, which corresponds to black-hole-type solutions.

Denote the deformation parameter by $\epsilon$ and assume that

$$
\epsilon>0 \text {. }
$$

The particular deformation considered will be seen to break the spherical symmetry (e.g., evenness in $Y$ ). The limit $\epsilon \rightarrow 0$ will be taken at the end of the calculation.

The two deformed metrics are, in fact, given by

$$
\begin{aligned}
g_{\mu \nu}^{ \pm}[T, X, Y, Z]= & {\left[\operatorname { d i a g } \left(-\left(1-2 M / \zeta^{ \pm}\right),\left(\zeta^{ \pm}\right)^{2} \sin ^{2} Z,\right.\right.} \\
& \left.\left.\left(1-2 M / \zeta^{ \pm}\right)^{-1}\left(d \zeta^{ \pm} / d Y\right)^{2},\left(\zeta^{ \pm}\right)^{2}\right)\right]_{\mu \nu}, \\
\zeta^{ \pm}= & \sqrt{b^{2}+Y^{2} \pm \epsilon Y b},
\end{aligned}
$$

for chart-2 coordinates without suffix ' 2 '. These two metrics are defined over two overlapping $Y$ domains:

$$
\begin{aligned}
& g_{\mu \nu}^{+} \text {for } Y \in(-\delta,+\infty), \\
& g_{\mu \nu}^{-} \text {for } Y \in(-\infty,+\delta),
\end{aligned}
$$


with an arbitrary value of $\delta$ in the open range $(0, \epsilon b / 2)$, for example

$$
\delta=\epsilon b / 4
$$

The Riemann tensors from these metrics are readily calculated and the corresponding Kretschmann scalars are found to be given by

$$
K^{ \pm}[Y]=48 \frac{M^{2}}{\left(b^{2}+Y^{2} \pm \epsilon Y b\right)^{3}} .
$$

With these results, we can define

$$
K[Y]=\left\{\begin{array}{l}
\lim _{\epsilon \rightarrow 0} K^{+}[Y] \text { for } Y \geq 0, \\
\lim _{\epsilon \rightarrow 0} K^{-}[Y] \text { for } Y<0,
\end{array}\right.
$$

which agrees with the previous result (3.5). The point of the above exercise is that $K$ has now been calculated with nonvanishing (invertible) metrics altogether.

\section{Appendix C. Nonsingular Solution in Different Coordinates}

In this appendix, we present the nonsingular black-hole solution from Sec. 47in terms of a different set of coordinates. The advantage of these new coordinates is that we only need one set of chart-2 coordinates and not two sets as used in (4.3) and (4.4).

Instead of starting from an Ansatz based on Kruskal-Szekeres coordinates as was done in our original article $\stackrel{11}{11}$ we start from an Ansatz based on Painlevé-Gullstrand (PG) coordinates $27 \mid 28$ (see, e.g., Ref. 29 for a brief review). Turning immediately to the chart-1 coordinates from Appendix A and replacing the radial coordinate $r$ of the PG coordinates by $\sqrt{b^{2}+\left(X_{1}\right)^{2}}$, we arrive at the following line element over part of the manifold (2.1):

$$
\begin{aligned}
\left.d s^{2}\right|_{\text {chart-1 }}= & -d \widehat{T}^{2}+\left(\frac{X_{1}}{\sqrt{b^{2}+\left(X_{1}\right)^{2}}} d X_{1}+\sqrt{\frac{2 M}{\sqrt{b^{2}+\left(X_{1}\right)^{2}}}} d \widehat{T}\right)^{2} \\
& +\left(b^{2}+\left(X_{1}\right)^{2}\right)\left(\left(d Z_{1}\right)^{2}+\left(\sin Z_{1}\right)^{2}\left(d Y_{1}\right)^{2}\right)
\end{aligned}
$$

for mass parameter $M>0$ and length parameter $b>0$. Remark that the surfaces of constant $\widehat{T}$ are intrinsically flat. ${ }^{29}$ An advantage of the metric (C.1a) is that it applies not only to the black-hole case $2 M \geq b$ (including the special case $2 M=b$ ) but also to the defect case $2 M<b$ 든

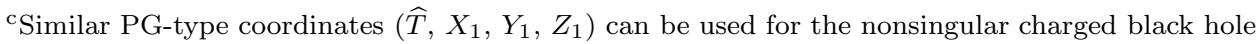
with the parameter choice $b \geq Q^{2} /(2 M)>0$, which differs from the choice $5.3 \mathrm{~b}$ considered in Sec. 5 The metric then takes the same form as the one from (C.1a) but with $2 M / \sqrt{b^{2}+\left(X_{1}\right)^{2}}$ replaced by $2 M / \sqrt{b^{2}+\left(X_{1}\right)^{2}}-Q^{2} /\left(b^{2}+\left(X_{1}\right)^{2}\right)$. For fixed positive values of $b$ and $M$, it is now possible to take the classical charge $|Q|$ arbitrarily small.
} 
We obtain the metrics for the $n=2$ and $n=3$ charts by taking the coordinates $\left(X_{2}, Y_{2}, Z_{2}\right)$ and $\left(X_{3}, Y_{3}, Z_{3}\right)$ instead of $\left(X_{1}, Y_{1}, Z_{1}\right)$. With the same replacements as used in (3.7), the corresponding metrics are given by the following line elements:

$$
\begin{aligned}
\left.d s^{2}\right|_{\text {chart-2 }}= & -d \widehat{T}^{2}+\left(\frac{Y_{2}}{\sqrt{b^{2}+\left(Y_{2}\right)^{2}}} d Y_{2}+\sqrt{\frac{2 M}{\sqrt{b^{2}+\left(Y_{2}\right)^{2}}}} d \widehat{T}\right)^{2} \\
& +\left(b^{2}+\left(Y_{2}\right)^{2}\right)\left(\left(d Z_{2}\right)^{2}+\left(\sin Z_{2}\right)^{2}\left(d X_{2}\right)^{2}\right), \\
\left.d s^{2}\right|_{\text {chart-3 }}= & -d \widehat{T}^{2}+\left(\frac{Z_{3}}{\sqrt{b^{2}+\left(Z_{3}\right)^{2}}} d Z_{3}+\sqrt{\frac{2 M}{\sqrt{b^{2}+\left(Z_{3}\right)^{2}}}} d \widehat{T}\right)^{2} \\
& +\left(b^{2}+\left(Z_{3}\right)^{2}\right)\left(\left(d Y_{3}\right)^{2}+\left(\sin Y_{3}\right)^{2}\left(d X_{3}\right)^{2}\right) .
\end{aligned}
$$

The PG coordinates play a special role in discussions of emergent gravity in superfluid systems ${ }^{30 \mid 31}$ Referring to (C.1), it appears, in principle, possible to have Unruh-type artificial black holes without curvature singularity. If such artificial black holes can indeed be realized with superfluids in the laboratory, the existence of closed time-like curves from the effective metric (last paragraph of Sec. 4) may signal the appearance of new topology-driven instabilities of the quasi-particle system.

\section{Appendix D. Weakened Elementary-Flatness Condition}

In this appendix, we take a closer look at the metric (5.5) near the defect core. In principle, it is possible to set $Q=0$ and even $M=Q=0$, as long as $b$ remains nonzero (here, taken to be positive).

Specifically, consider a small neighbourhood around the spacetime point $P$ with the following chart-2 coordinates:

$$
\left.\left(T, X_{2}, Y_{2}, Z_{2}\right)\right|_{P}=(0,0,0, \pi / 2) \text {. }
$$

Next, define dimensional coordinates $(t, \widetilde{x}, y, \widetilde{z})$ which vanish at $P$ :

$$
\left(T, X_{2}, Y_{2}, Z_{2}\right)=(0,0,0, \pi / 2)+(t, \widetilde{x} / b, y, \widetilde{z} / b) .
$$

The metric (5.5) near $P$ then becomes

$$
\begin{aligned}
\left.d s^{2}\right|_{\text {near }-P}= & -\left(1-\frac{2 M}{\sqrt{b^{2}+y^{2}}}+\frac{Q^{2}}{b^{2}+y^{2}}\right) d t^{2} \\
& +\left(1-\frac{2 M}{\sqrt{b^{2}+y^{2}}}+\frac{Q^{2}}{b^{2}+y^{2}}\right)^{-1} \frac{y^{2}}{b^{2}+y^{2}} d y^{2} \\
& +\left(1+y^{2} / b^{2}\right)\left(d \widetilde{z}^{2}+\left[1+O\left(\widetilde{z}^{2} / b^{2}\right)\right] d \widetilde{x}^{2}\right) \\
\sim & -\left(1-2 M / b+Q^{2} / b^{2}\right) d t^{2} \\
& +\left(1-2 M / b+Q^{2} / b^{2}\right)^{-1} \frac{y^{2}}{b^{2}} d y^{2}+d \widetilde{z}^{2}+d \widetilde{x}^{2} .
\end{aligned}
$$


With a further change of coordinates,

$$
\begin{aligned}
& \widetilde{t}=A t, \\
& \widetilde{y}=\left\{\begin{array}{l}
+y^{2} /(2 b A) \text { for } y \geq 0, \\
-y^{2} /(2 b A) \text { for } y<0,
\end{array}\right. \\
& A \equiv \sqrt{1-2 M / b+Q^{2} / b^{2}}>0,
\end{aligned}
$$

the metric near $P$ reads

$$
\left.d s^{2}\right|_{\text {near-P }} \sim-d \widetilde{t}^{2}+d \widetilde{y}^{2}+d \widetilde{z}^{2}+d \widetilde{x}^{2} .
$$

which corresponds to a patch of Minkowski spacetime.

Observe that the coordinate transformation (D.4b) is a $C^{1}$ function with a discontinuous second derivative at $y=0$. That is, the coordinate transformation is not a diffeomorphism, which is a $C^{\infty}$ function everywhere. The standard elementaryflatness condition relies, however, on genuine diffeomorphisms. Hence, the metric (5.5) obeys a weakened version of the elementary-flatness condition, allowing for non-smooth coordinate transformations [the same conclusion holds for the metrics (C.1) from Painlevé-Gullstrand-type coordinates]. In other words, the spacetime does not correspond to a Lorentzian manifold d Whether or not such spacetimes play a role in classical physics may ultimately be up to quantum gravity to decide.

\section{Bibliography}

1. K. Schwarzschild, "Über das Gravitationsfeld eines Massenpunktes nach der Einsteinschen Theorie," Sitzungsberichte der Deutschen Akademie der Wissenschaften zu Berlin, Klasse für Mathematik, Physik, und Technik (1916), pp. 189-196 [scanned version available from http://de.wikisource.org].

2. S.W. Hawking and G.F.R. Ellis, The Large Scale Structure of Space-Time (Cambridge Univ. Press, Cambridge, England, 1973).

3. C.W. Misner, K.S. Thorne, and J.A. Wheeler, Gravitation (Freeman, New York, 1973).

4. H.A. Bethe, "Theory of diffraction by small holes," Phys. Rev. 66, 163 (1944).

5. S. Bernadotte and F.R. Klinkhamer, "Bounds on length scales of classical spacetime foam models," Phys. Rev. D 75, 024028 (2007), arXiv:hep-ph/0610216

6. F.R. Klinkhamer and M. Schreck, "New two-sided bound on the isotropic Lorentzviolating parameter of modified Maxwell theory," Phys. Rev. D 78, 085026 (2008), arXiv:0809.3217.

7. M. Schwarz, Nontrivial Spacetime Topology, Modified Dispersion Relations, and an SO(3)-Skyrme Model, PhD Thesis, KIT, July 2010 (Verlag Dr. Hut, München, Germany, 2010).

8. F.R. Klinkhamer and C. Rahmede, "Nonsingular spacetime defect," Phys. Rev. D 89, 084064 (2014), arXiv:1303.7219.

\footnotetext{
${ }^{\mathrm{d}}$ The solutions of the Klein-Gordon equation over the nonsingular defect manifold with the $M=Q=0$ metric (5.5) differ from those of Minkowski spacetime 10
} 
9. F.R. Klinkhamer, "Skyrmion spacetime defect," arXiv:1402.7048

10. F.R. Klinkhamer and F. Sorba, "Comparison of spacetime defects which are homeomorphic but not diffeomorphic," arXiv:1404.2901

11. F.R. Klinkhamer, "Black-hole solution without curvature singularity," Mod. Phys. Lett. A 28, 1350136 (2013), arXiv:1304.2305

12. F.R. Klinkhamer, "Black-hole solution without curvature singularity and closed timelike curves," Acta Phys. Pol. B 45, 5 (2014) arXiv:1305.2875.

13. F.R. Klinkhamer, "A 'regularized' Schwarzschild solution," to appear in: Proceedings of the Karl Schwarzschild Meeting on Gravitational Physics (Frankfurt Institute for Advanced Studies, July 2013), preprint KA-TP-23-2013 [available from http://www.itp.kit.edu ].

14. E. Ayon-Beato and A. Garcia, "Regular black hole in general relativity coupled to nonlinear electrodynamics," Phys. Rev. Lett. 80, 5056 (1998), arXiv:gr-qc/9911046.

15. A. Garcia, E. Hackmann, J. Kunz, C. Lämmerzahl, and A. Macias, "Motion of test particles in a regular black hole space-time," arXiv:1306.2549.

16. E.W. Weisstein, "Boy Surface," from MathWorld - A Wolfram Web Resource http://mathworld.wolfram.com/BoySurface.html.

17. M.D. Kruskal, "Maximal extension of Schwarzschild metric," Phys. Rev. 119, 1743 (1960).

18. G. Szekeres, "On the singularities of a Riemannian manifold," Publ. Math. Debrecen 7, 285 (1960).

19. V.A. Kostelecky and R. Lehnert, "Stability, causality, and Lorentz and CPT violation," Phys. Rev. D 63, 065008 (2001), arXiv:hep-th/0012060

20. C. Adam and F. R. Klinkhamer, "Causality and CPT violation from an Abelian Chern-Simons like term," Nucl. Phys. B 607, 247 (2001), arXiv:hep-ph/0101087

21. H. Reissner, "Über die Eigengravitation des elektrischen Feldes nach der Einsteinschen Theorie," Annalen der Physik 50, 106 (1916).

22. G. Nordström, "On the energy of the gravitational field in Einstein's theory," Proceedings Academy of Sciences of Amsterdam 26, 1201 (1918).

23. J.C. Graves and D.R. Brill, "Oscillatory character of Reissner-Nordstrom metric for an ideal charged wormhole," Phys. Rev. 120, 1507 (1960).

24. B. Carter, "The complete analytic extension of the Reissner-Nordström metric in the special case $e^{2}=m^{2}, "$ Phys. Lett. 21, 423 (1966).

25. M. Visser, Lorentzian Wormholes: From Einstein to Hawking (Springer, New York, 1995).

26. K.S. Virbhadra and G.F.R. Ellis, "Gravitational lensing by naked singularities," Phys. Rev. D 65, 103004 (2002).

27. P. Painlevé, "La mécanique classique et la théorie de la relativité," C. R. Acad. Sci. (Paris) 173, 677 (1921).

28. A. Gullstrand, "Allgemeine Lösung des statischen Einkörper-problems in der Einsteinschen Gravitationstheorie," Arkiv. Mat. Astron. Fys. 16, 1 (1922).

29. K. Martel and E. Poisson, "Regular coordinate systems for Schwarzschild and other spherical space-times," Am. J. Phys. 69, 476 (2001), arXiv:gr-qc/0001069

30. W.G. Unruh, "Experimental black hole evaporation," Phys. Rev. Lett. 46, 1351 (1981).

31. G.E. Volovik, The Universe in a Helium Droplet, paperback edition (Oxford Univ. Press, Oxford, England, 2009). 